\title{
Radical pair recombination stereoselectivity as a probe of magnetic isotope and magnetic field effects
}

\author{
George Lem and Nicholas J. Turro* \\ Chemistry Department, Columbia University, New York, New York 10027, USA. E-mail: turro@chem.columbia.edu
}

Received (in Corvallis, OR, USA) 22nd November 1999, Accepted 7th January 2000

\begin{abstract}
Photolysis of meso- or $d l$-2,4-diphenylpentan-3-one within $\mathrm{NaY}$ zeolites, coadsorbed with a chiral inductor, lead to enantioselective radical pair recombinations which are sensitive to ${ }^{13} \mathrm{C}$ isotope effects, but insensitive towards external magnetic field effects.
\end{abstract}

We report here rationally designed systems, based on wellestablished radical pair chemistry, which investigate the stereoselectivity of recombination of prochiral radical pairs for which significant magnetic field effects and magnetic isotope effects have been observed in micelles. ${ }^{1,2}$ The concept of the systems is the following. A prochiral triplet radical pair that can be produced reversibly is generated in a chiral, supramolecular ${ }^{3}$ environment that (i) allows the separation of the pair to distances allowing for the exchange interaction between the partners of the pair to decrease to values of the order smaller than those available from external or internal magnetic fields, ${ }^{1}$ and (ii) encourages reencounters of the pair. Each recombination of the pair can produce either the $(+)$ or $(-)$-enantiomer, but in the presence of a chiral inductor within the supramolecular environment, a certain enantiomeric selectivity can result. ${ }^{4}$ For any given system in which some ee is observed, the efficiency of ee resulting from radical pair recombination is expected to depend on the radical pair lifetime, which has consequences in terms of the effective interactions between the pair and the chiral inductor. In this scenario, magnetic field and magnetic isotope effects on the enantioselectivity may result if the lifetime of the pair is extended or diminished. From a survey of published results, we selected the supercage of $\mathrm{NaY}$ faujasite zeolites $^{5}$ as the host supramolecular environment, ${ }^{6}$ the photolyses of meso- and $d l$-2,4-diphenylpentan-3-one (meso-1 and $d l$ $\mathbf{1}$, respectively) to generate triplet radical pairs and a coadsorbed chiral inductor such as diethyl L-tartrate and (+)- or $(-)$-ephedrine as chiral inductors.

As depicted in Scheme 1, photolysis of meso-1 results in the formation of $d l-\mathbf{1}$ and vice versa (for simplicity only the photoprocesses of meso-1 are shown). ${ }^{7}$ According to the conventional paradigm of ketone photochemistry, ${ }^{8} \alpha$-cleavage of triplet excited 1 leads to the formation of sec-phenethyl and $s e c$-phenethylacyl triplet geminate radical pairs (GRP). If the space available in the supercage allows rapid diffusional separation of the primary geminate pair, then decarbonylation of the sec-phenethylacyl radical and subsequent formation of diastereomeric 2,3-diphenylbutanes 2 occurs. ${ }^{7}$ If diffusion is restricted, but rotation of the triplet radical pairs relative to one another is permitted, intersystem crossing (ISC) of the radical pairs occurs to yield meso-1 or $d l-1$. Additionally, if the environment is chiral, $d l-\mathbf{1}$ with some degree of enantiomeric enrichment can be produced. Of these processes, only the rate of ISC is sensitive towards magnetic effects. ${ }^{1 a, b}$ Thus, the ratios between decarbonylation products and recombination products, ${ }^{2 c}$ as well as the ee of photoproduced $d l-\mathbf{1}$ can serve as probes for magnetic effects on the radical pair recombination stereochemistry. While we expect the ratio of recombination products/decarbonylation products to increase as the rate of ISC increases, predictions on the direction of change of the ee of photoproduced $d l-\mathbf{1}$ cannot be made a priori.

Table 1 summarizes the ee values of recovered $d l-\mathbf{1}$ upon photolysis of $d l-\mathbf{1}$ in NaY co-loaded with diethyl L-tartrate as a function of both external magnetic field strength and isotope. It can be seen that there are no differences, within experimental error, in the ee (measured by chiral GC) of the photolyzed $d l-1$ when the photoreaction is carried out at either 0 or $2 \mathrm{kG}$. However, upon photolysis at $0 \mathrm{kG}$ of $d l-1$ enriched in ${ }^{13} \mathrm{C}(98 \%$ enriched) at the carbonyl carbon, a significant increase in the ee of the recovered $d l-1$ is observed when the ${ }^{13} \mathrm{C}$ labeled isomer is photolyzed relative to the unlabeled isotopomer (3.0 vs. $1.6 \%$, respectively). Additionally, a slight increase in the amount of recombination product $v s$. decarbonylation products was observed in the photolysis of $d l-1-{ }^{13} \mathrm{CO}$ relative to $d l-1-{ }^{12} \mathrm{CO} .^{9}$ However, no such trend has seen in the photolysis of $d l-1-{ }^{12} \mathrm{CO}$ performed at 0 vs. $2 \mathrm{kG}$.

In order to assess the influence of initial stereochemistry of $\mathbf{1}$ on the enantioselectivity of $d l-\mathbf{1}$ as a function of external magnetic field and isotope effects, photolyses of meso-1 were performed in $\mathrm{NaY}$ using ephedrine as the chiral inductor. ${ }^{10}$ From Table 2, it can be seen that photolyses of meso-1 in NaY in the presence of ephedrine, significantly higher ees for $d l-\mathbf{1}$ are obtained compared to utilizing $d l \mathbf{- 1}$ as the starting material and diethyl L-tartrate as the inductor. However, similar to the results obtained with diethyl L-tartrate, only a ${ }^{13} \mathrm{C}$ isotope effect is observed while external field effects are absent. Thus, an

Table 1 Photolysis of $d l-\mathbf{1}$ in NaY co-loaded with diethyl tartrate ${ }^{a}$

\begin{tabular}{llll}
\hline Magnetic field $/ \mathrm{kG}^{b}$ & Ketone & Chiral inductor & Ee of $d l-\mathbf{1}(\%)^{c d}$ \\
\hline 2 & $d l-\mathbf{1}^{-12} \mathrm{CO}$ & diethyl L-tartrate & $+2.0 \pm 0.2$ \\
0 & $d l-\mathbf{1}^{-12} \mathrm{CO}$ & diethyl L-tartrate & $+1.6 \pm 0.3$ \\
0 & $d l-\mathbf{1}^{-13} \mathrm{CO}$ & diethyl L-tartrate & $+3.0 \pm 0.1$
\end{tabular}

${ }^{a}$ The average occupancy of $d l-\mathbf{1}$ in $\mathrm{NaY}$ is $\sim 0.05$ molecules per cage and that of diethyl tartrate $\sim 1$ molecules per cage; $d l-\mathbf{1}$ was photolyzed to $35-45 \%$ conversion. ${ }^{b}$ Ref. $12 .{ }^{c}$ Errors correspond to standard deviations derived from three or more independent experiments. ${ }^{d}$ After photolysis, $d l$ $\mathbf{1}$ is separated from meso-1 via preparative TLC and analyzed by chiral GC.

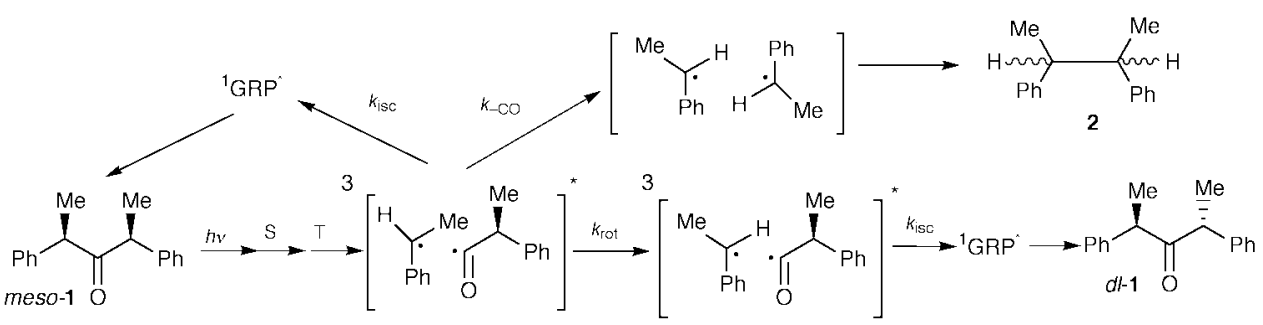

Scheme 1 
Table 2 Photolysis of meso-1 in NaY co-loaded with ephedrine ${ }^{a}$

\begin{tabular}{|c|c|c|c|}
\hline Magnetic field/kG ${ }^{b}$ & Ketone & Chiral inductor & Ee of $d l-1(\%)^{c d}$ \\
\hline 2 & meso-1-12 CO & (-)-ephedrine & $+4.9 \pm 1.9$ \\
\hline 0 & meso-1-12 CO & (-)-ephedrine & $+4.7 \pm 1.9$ \\
\hline 0 & meso-1-13 CO & (-)-ephedrine & $+9.9 \pm 1.3$ \\
\hline 2 & meso-1-12 CO & $(+)$-ephedrine $e$ & $-4.3 \pm 1.6$ \\
\hline 0 & meso-1-12 CO & $(+)$-ephedrine $e$ & $-3.6 \pm 0.6$ \\
\hline 0 & meso-1-13 $\mathrm{CO}$ & $(+)$-ephedrine $e$ & $-7.1 \pm 0.5$ \\
\hline
\end{tabular}

$a$ The average occupancy of meso- $\mathbf{1}$ in NaY is $\sim 0.05$ molecules per cage and that of ephedrine $\sim 1$ molecules per cage; meso-1 was photolyzed to 26-50\% conversion. ${ }^{b}$ Ref. 12. ${ }^{c}$ Errors correspond to standard deviations derived from three independent experiments. ${ }^{d}$ After the photolysis, $d l-\mathbf{1}$ is separated from meso-1 via preparative TLC and analyzed by chiral GC. $e$ The hemihydrate form was used.

approximate two-fold increase in the ee of $d l-\mathbf{1}$ is observed upon photolysis of meso-1-13 CO compared to that of meso-1-12 $\mathrm{CO}$ [+10\% vs. $+5 \%$, with (-)-ephedrine] (Table 2). Additionally, more recombination product was observed for the photolysis of meso-1-13 CO compared to that of meso-1-12 CO. ${ }^{11}$

We can interpret the data in Tables 1 and 2 based on the mechanism of triplet-singlet ISC for triplet biradicals. ${ }^{13,2 c, f}$ It is recognized that if triplet radical pairs are separated by distances of several $\AA$ or more, the singlet-triplet energy gap is decreased and ISC is controlled by weak hyperfine couplings induced by nuclear-spin interactions which are magnetic field and isotope dependent. Thus, ${ }^{13} \mathrm{C}$ is expected to induce a faster ISC in the radical pair relative to ${ }^{12} \mathrm{C}$, for which only protons provide a hyperfine mechanism for ISC. External magnetic fields can reduce the rate of ISC by increasing the S-T energy gap for two of the three triplet sublevels. Experimental results for such phenomena have been the subject of several reviews. ${ }^{1,2}$

In hydrocarbon solutions, sec-phenethylacyl radicals decarbonylate with a rate constant of $\sim 5 \times 10^{7} \mathrm{~s}^{-1} .^{14}$ In polar solvents, the rate of CO loss can be reduced. ${ }^{15}$ Since the internal cages of zeolite supercages are considered highly polar, ${ }^{16}$ decarbonylation in the zeolite must be significantly slower than $5 \times 10^{7} \mathrm{~s}^{-1}$. Recombination of structurally related benzoyl and cumyl radical pairs obtained from the photolysis of dimethyldeoxybenzoin, in SDS micellar solutions, has been measured to occur with a rate constant on the order of $\sim 1.2 \times 10^{7} \mathrm{~s}^{-1} .17$ This rate is expected to be increased by ${ }^{13} \mathrm{C}$ isotope effects, via changes in the rate of ISC. An increased rate of ISC can be manifested in the different ee values for recovered $d l$-1 obtained upon irradiation of either meso- or $d l-1$ containing different isotopic labels at the carbonyl carbon. Since there is a lack of information in the rotational dynamics between the prochiral GRPs and the chiral inductor, the trend of the ee of the photoreaction cannot be predicted a priori.

The experimental results show an increase in the ee of $d l$ $1-{ }^{13} \mathrm{CO}$ relative to its ${ }^{12} \mathrm{C}$ isotopomer. Furthermore, photolysis of $1^{13} \mathrm{CO}$ leads to a slight decrease in the amount of decarbonylated products relative to the ${ }^{12} \mathrm{C}$ labeled isotopomer. ${ }^{9,11}$ Similar results have also been previously observed for the photolysis of dibenzylketone- ${ }^{13} \mathrm{CO}$ in faujasites. ${ }^{2 c}$ Hence, an increase in the ee of the photoproduced $d l-1-13 \mathrm{CO}$ may be a function of its faster rate of ISC, leading to a greater number of recombinational processes from the primary GRP as compared to $d l-1-{ }^{12} \mathrm{CO}$ which maintains a relatively lower rate of ISC from the triplet GRP and thus higher propensity to form decarbonylated products. ${ }^{2 a, b, 4 a, 18}$

According to the product ratios and the ee values expressed in Tables 1 and 2, there are no observable external field effects in the photolysis of either meso- or $d l-1$. At this time we lack a theoretical kinetic model to explain these experimental results. Although it has been widely demonstrated that magnetic field and magnetic isotope effects can influence the rate of ISC for triplet biradicals in opposite manners, there is a lack of information on the magnitudes of those rates. It can be argued that external field effects, as they pertain to $\mathbf{1}$ in faujasites, are very small and subtle as compared to magnetic isotope effects. Indeed, in similar product studies done with dibenzyl ketone
(DBK) adsorbed in faujasites, external magnetic field effects were significantly smaller than those observed for ${ }^{13} \mathrm{C}$ isotope effects. ${ }^{2 c}$

The results show that photochemical enantiomeric selectivity through geminate radical recombinations in zeolite cavities is feasible. The most important finding is that ee values are sensitive to magnetic isotope effects, and consequently can be used as a probe of radical pair dynamics in zeolites.

This work was supported in part by the NSF and the Department of Energy under Grant No. NSF CHE 9810367 to the Environmental Molecular Sciences Institute at Columbia University and National Science Foundation Grant No. CHE 98-12676. We are especially indebted to Dr Valery Tarasov of the Russian Academy of Sciences for helpful discussions and sharing unpublished data.

\section{Notes and references}

1 (a) U. E. Steiner and T. Ulrich, Chem. Rev., 1989, 89, 51; (b) K. M. Salikhov, Yu. N. Molin, R. Z. Sagdeev and A. L. Buchachenko, in Spin Polarization and Magnetic Effects in Radical Reactions, Elsevier, Amsterdam, 1984; (c) N. J. Turro, Proc. Natl. Acad. Sci. U.S.A., 1983, 80, 609; (d) N. J. Turro and B. Kraeutler, Acc. Chem. Res., 1980, 13, 369.

2 (a) E. N. Step, V. F. Tarasov, A. L. Buchachenko and N. J. Turro, J. Phys. Chem., 1993, 97, 363; (b) V. Tarasov, N. D. Ghatlia, A. L. Buchachenko and N. J. Turro, J. Phys. Chem., 1991, 95, 10220; (c) N. J. Turro and Z. Zhang, Tetrahedron Lett., 1989, 30, 3761; (d) N. J. Turro, M. B. Zimmt and I. R. Gould, J. Phys. Chem., 1988, 92, 433; (e) M. B. Zimmt, C. Doubleday and N. J. Turro, J. Am. Chem. Soc., 1985, 107, 6727; (f) M. B. Zimmt, C. Doubleday and N. J. Turro, J. Am. Chem.Soc., 1984, 106, 3363; (g) N. J. Turro and G. C. Weed, J. Am. Chem. Soc., 1983, 105, 1861; (h) B. H. Baretz and N. J. Turro, J. Am. Chem. Soc., 1983, 105, 1309.

3 J.-M. Lehn, in Supramolecular Chemistry, VCH, Weinheim, 1995; V. Balzani and F. Scandola, in Supramolecular Photochemistry, PrenticeHall, New York, 1991, ch. 3.

4 N. A. Kaprinidis, M. S. Landis and N. J. Turro, Tetrahedron Lett., 1997, 38, 2609; V. J. Rao, S. R. Uppili, D. R. Corbin, S. Schwarz, S. R. Lustig and V. Ramamurthy, J. Am. Chem. Soc., 1998, 120, 2480; A. Joy, R. J. Robbins, K. Pitchumani and V. Ramamurthy, Tetrahedron Lett., 1997, 38, 8825; G. Sundarababu, M. Leibovitch, D. R. Corbin, J. R. Scheffer and V. Ramamurthy, Chem. Commun., 1996, 2159.

5 D. W. Breck, in Zeolite Molecular Sieves: Structure, Chemistry and Use, Wiley, New York, 1974.

6 V. Ramamurthy, in Photochemistry in Organized and Constrained Media, ed. V. Ramamurthy, VCH, New York, 1991.

7 N. D. Ghatlia and N. J. Turro, J. Photochem. Photobiol. A: Chem., 1991, 57, 7 .

8 N. J. Turro, in Modern Molecular Photochemistry, BenjaminCummings, Menlo Park, CA, 1978, ch. 13.

9 Ratio of meso-1:2 $=0.12$ for the photolysis of $d l-1-12 \mathrm{CO}$, ratio of meso-1:2 $=0.19$ for the photolysis of $d l-1-13 \mathrm{CO}$.

10 No data is reported for the stereoselectivity of the photoreaction of meso-1 in NaY-diethyl tartrate systems due to analytical difficulties in measuring the ee of $d l-1$ recovered from such photolysis.

11 Ratio of $d l-\mathbf{1}: \mathbf{2}=0.64$ for the photolysis of meso-1-12 CO, ratio of $d l$ $\mathbf{1}: \mathbf{2}=0.92$ for the photolysis of meso-1-13 CO.

12 Irradiations at $2 \mathrm{kG}$ were performed by photolyzing the sample between the poles of a permanent magnet.

13 N. J. Turro, in Modern Molecular Photochemistry, Benjamin-Cummings, Menlo Park, CA, 1978, ch. 6.

14 I. R. Gould, B. H. Baretz and N. J. Turro, J. Phys. Chem., 1987, 91, 925; N. J. Turro, I. R. Gould and B. H. Baretz, J. Phys. Chem., 1983, 87, 531.

15 Y. P. Tsentalovich and H. Fisher, J. Chem. Soc., Perkin Trans. 2, 1994, 729.

16 V. Ramamurthy, D. R. Sanderson and D. F. Eaton, Photochem. Photobiol., 1992, 56, 29; K. K. Iu and J. K. Thomas, Langmuir, 1990, 6, 471.

17 I. R. Gould, M. B. Zimmt, N. J. Turro, B. H. Baretz and G. F. Lehr, J. Am. Chem. Soc., 1985, 107, 4605.

18 V. F. Tarasov, N. D. Ghatlia, A. L. Buchachenko and N. J. Turro, J. Am. Chem. Soc., 1992, 14, 9517; V. F. Tarasov, N. D. Ghatlia, N. I. Avdievich, I. A. Shkrob, A. L. Buchachenko and N. J. Turro, J. Am. Chem. Soc., 1992, 116, 2281; V. F. Tarasov, I. A. Shkrob and A. L. Buchachenko, Chem. Phys. Lett., 1989, 135, 391. 